\title{
The Technical Buzzword: Artificial Intelligence
}

\author{
Banya Arabi Sahoo
}

\begin{abstract}
AI is the incredibly exciting technique to the world. According to John McCarthy it is "The science and engineering of making intelligent machine, especially intelligent computers". AI is the way of creating extraordinary powerful machine which is similar as human being. The AI is being accomplished by studying how human brain think, how they learn, decide, work, solving the real world problem and after that verify the outcomes and studying it. Primarily you can learn here what AI is and how it works, its types, its history, its agents, its applications, its advantages and disadvantages.
\end{abstract}

Keywords: "The science and engineering of making intelligent machine, especially intelligent computers".

\section{INTRODUCTION}

The passion of the young generation is always being in trending. Artificial Intelligence is also one of the trends now. We are creating a brain map with the help of AI. The AI is technology to create a machine which makes the impossible to possible, whatever a person can think this AI can make it possible. A creature that can think like a human behave like human and also intelligent like human being. Otherwise, we can call it Machine Intelligence. The artificial Intelligence was coined by John McCarthy in 1976.

\section{WHAT IS INTELLIGENCE}

Intelligence is natural. It defers persons to persons. What it is actually : It is the ability to calculate, learn from experience, solve the problems, getting the ideas, classify, generalize, adopt a new situation, store and retrieve information etc. If its human being then its human intelligence or if it machines then its machine intelligence. Only a machine can equipped almost all intelligence in it.

\section{AIM BEHIND THE AI} can be implemented in every real world problem to fix it. The expert system that expert in many categories like behavior, learning, demonstration , explanation and can give valuable advice to user and solve lots of accidental causes. It is combination of thinking capacity of a human and intelligence of a machine and program. To save the cost optimization processes many companies are getting profit because of AI.

\footnotetext{
Manuscript received on 13 April 2021 | Revised Manuscript received on 28 June, 2021| Manuscript Accepted on 15 August 2021 | Manuscript published on 30 August 2021. * Correspondence Author

Banya Arabi Sahoo*, Teacher, Department of Computer Science, Dav public school, Chandrasekharpur, Bhubaneswar (Odisha), India.

(C) The Authors. Published by Lattice Science Publication (LSP). This is an open access article under the CC-BY-NC-ND license (http://creativecommons.org/licenses/by-nc-nd/4.0/)
}

The arrow point of AI is to create an expert system that

\section{HISTORY OF AI}

The first ever concept of AI was coming from Greek mythology . This machine intelligence concept was started around at that time, but recently it was become a technical buzzword. The first pioneer project conceived on 1950s.

1950s : Turing Test :

The great mathematician Alan Turing find out the ability of thinking capacity of a machine is equivalent to human being.

\section{5s: Buzzword AI :}

The word AI coined in 1955. The American scientist John McCarthy first use term of AI in Dartmouth college

There they discussed that how machine could be made of use language, solve the problem as human being.

\section{6 - Eliza the Chatbot:}

Eliza is the first ever chatbot. Its eventually search for the word of its synonyms for the sentence entered via keyboard by the user. This communication based on like a searching in a thesaurus. It's the early implementation of Turing test.

1970 s to 1980s: AI winter:

In this period no new concept or any research were developed. So, it's called the winter of AI .

\section{7: Deep Blue:}

This deep blue is a chess player computer developed by IBM. It's the first chess match and game. Its actually started the fear that may be a machine could prevail over human.

\section{1: Siri}

In 2011 IPhone produces a personal assistant which process natural language. This software recognizes the entry try the user and work on it.

\section{1: Watson}

Another super computer named Watson was developed by IBM. Its founded by Thomas J Watson. Its own an American quiz show. This was able to answer the question in natural spoken language. It is so big with full of hardware and data storage devices which looks like 10 refrigerators.

\section{4: Alexa}

This is the most popular and intelligent language based assistant. This can be use anywhere kitchen, bedroom, living room for a lot of people.

In the mediaeval time a tay-chatbot was developed by Microsoft but its being banned later. Then another alpha-golike chess game deep blue was developed by Google.

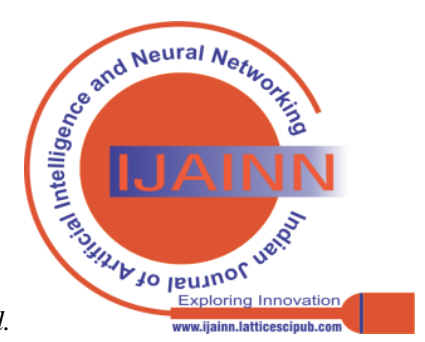




\section{TYPES OF AI}

1. Expert system

2. Fuzzy logic

3. Neural network

4. Robotics

\section{Expert System:}

AN expert system is the concept of behavior . It is a system that imitates the human behavior for example : Decision making, reasoning solve the complex problem. It is expertise is every field of human behavior. Expert system consist of different elements like user interface, inference engine and knowledge base etc. The user interface part is behave as intermediate in between user of the expert system and expert system.

\section{Fuzzy Logic:}

Fuzzy logic is defined as the concept of human thinking of taking decision in any type of situation. It make the decision as possibilities of yes/no or true /false . its takes the decision logically as input and produces the output in the form of true/false . it purely work on possibilities . it has four main parts .
i. Fuzzification module.
ii. Knowledge base inference engine.
iii. Defuzzification module.

\section{Neural Network:}

Neural network works like our brain. It imitates the real network of human being. The ANN (Artificial neural network) following the working of human brain. Our brain consist of millions of nerve cells known as neurons. Those neurons are connected to other neurons by axon and a neuron send the data in to different neurons. In ANN , neurons are behave as biological neurons and those nodes are connected to each other by links for communication or interaction. Those nodes accept the data and perform different operation and produces output as node value.

\section{Robotics:}

Robotics is the important part of AI. A robot is an artificial agent that works in the real world environment and deal with surroundings. It is the recreation of human thinking process simply called it's a manmade machine with human's intellectual abilities.

\section{AGENT AND ENVIRONMENT OF AI}

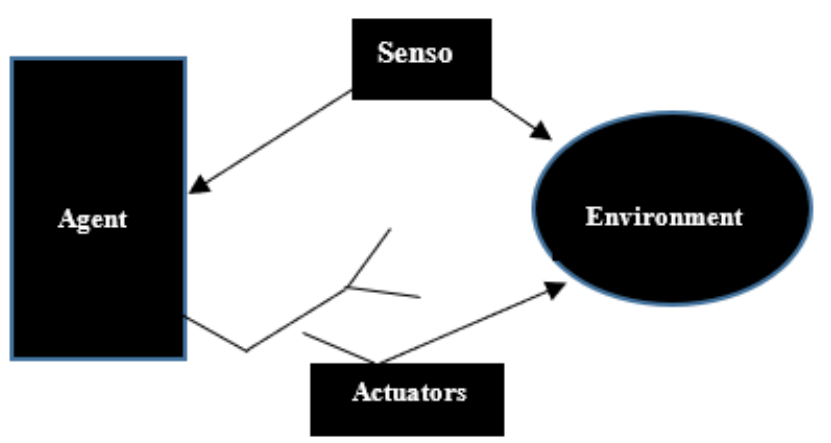

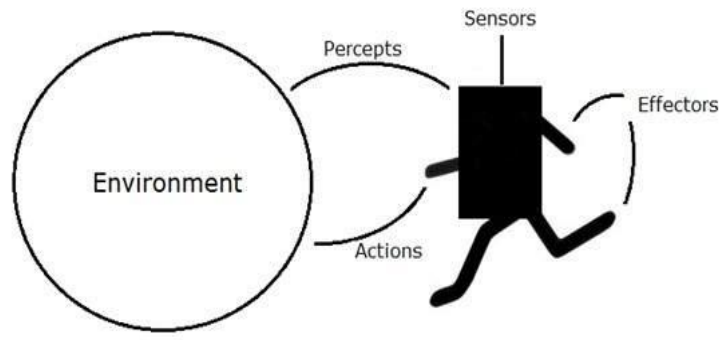

AI is the study of rational agents. An agent could be anything which makes decision likes person, firm machine or software. An AI system is the combination of agent and its environment. An agent is something that can sense its environment through effectors. The agent will take the appropriate decision after considering the outcomes. The AI system consist of agents and environment. An agent receive the signal about environment through sensors and take action on environment through actuators.

\section{Agent $=$ Architecture + Agent program}

An agent execute on a machinery object which called architecture for example camera, a pc, robotic vehicle etc. An agent program which controlling the agent function . functions are like map where the sequence of actions stored.

\section{EXAMPLE OF AI}

* Siri in iphone and ipad , Tesla ,Cogito ,Netflix, Cortana, Online customer support , Face recognition/ search, Social media sites, Pandora,Video games ,Smart home devices, drones etc. these are the basic example of AI that we use in daily life.

* Far apart we have video game that we using our day to day life. Its also uses the AI for its physical motions.

* The airplanes/space shuttles are also using AI for the safety of people.

* In our daily life we all are facing a lots of problems due to a bad comments or tweets .so to control this positive and negative flow of social media messages AI is being used. That's called sentimental search engine. For example :www.clevly.com

* AI also used for analyzed the data.

* Its also predict the stock market and whether forecasts.

* Its solve the problem of shape recognition and image manipulation.

\section{APPLICATION OF AI}

- Deep learning application

- $\quad$ Predictive analytics

- Translation

- Classification and clustering

- Information extraction

- Speech to text/ text to speech 
- Image recognition

- Machine vision

- $\quad$ Planning, scheduling and optimization

- Robotics \& soft robotics

- Expert system

- Health care

- Visualization

- Simulation modeling

- Natural learning process

\section{Artificial Intelligence in Healthcare:}

Machine learning is applied by many companies for better and faster diagnosis and treatment availability checking than human being. In my above paragraph "history of AI" I have define about Watson which is developed about by IBM. Its one AI which understand the natural language and can respond the question frequently. This technology determine the patient data and other available data regarding the patient from different sources for faster recovery. Its integrating medical activities ,software and cognitive science also helpful in future scientific medical discipline.

\section{Artificial Intelligence in business:}

The best of AI technology is Robotics. This robotic automation is highly using in different business sectors.. which reduces the repetitive task of human being. The machine learning technique is used in CRM (Customer relationship management) which is actually work how to better serve to the customer. Another AI called chatbot which is incorporated din to websites and e-companies for immediate service of customers.

\section{AI in education:}

AI helps in education sectors like access student records, adopt their needs, helping them work at their own pace , gradation and report can easily done.

\section{AI in Autonomous vehicles:}

A vehicle having different activities which is done by a human. When a machine responsible for driving a vehicle that is called self-driving vehicle. It has some sensors, which choose specific action according to the situations. Some of the tools are like long range radar, cameras, lidar etc. All those different tools having different capacities. They use to collect information and taking the actions as like human being.

Several application :

- If vehicle running low fuel then it search and directing towards gas station for fuel.

- Finding out the quickest route according to the traffic conditions.

- By using speed recognition it communicate with the passengers.

- $\quad$ The AI feature virtual assistance like siri, alexa we can use here as natural language interface.

\section{AI for robotics}

It's the best gift of AI to the world. It reduces the human interactions but helpful in many things like bring down the traffic accidents and deaths, enable disaster response for dangerous situations forexample : nuclear meltdown at the Fukushima power plant.

\section{ADVANTAGES AND DISADVANTAGES OF AI}

\section{Advantages:}

- $\quad$ It reduces the error.

- More powerful and more use of computers

- Increasing work efficiency and solving new problems.

- Daily applications with digital assistant

- Improved interface as well as better handling of information.

\section{Disadvantages:}

- High cost

- Lesser jobs

- Lack of personal connection and less experienced programmers.

- Addiction

- $\quad$ Efficient decision making

\section{CONCLUSION}

Artificial Intelligence is so productive as well as growing at quicker pace. Its changing our day to day life, influencing the way we live, interact. Everyday the we go far and far to bring some changes. There is much more to come in the coming years with more improvements, development, and governance. AI to makes discoveries, generating new ideas, conduct experiments. The AI will create more advance AI day to day.

\section{ACKNOWLEDGMENT}

The author would like to thanks.

\section{REFERENCE}

1. https://www.researchgate.net/publication/323498156_Artificial_Intel ligence

2. https://blog.solvatio.com/en/from-deep-blue-to-alexa-the-history-ofartificial-intelligence

3. https://www.geeksforgeeks.org/agents-artificial-intelligence/

4. https://www.tutorialspoint.com/artificial_intelligence/artificial_intell igence_agents_and_environments.htm

5. https://www.researchgate.net/publication/325581483_Introduction_t o Artificial Intelligence

6. https://www.dcpehvpm.org/E-Content/BCA/BCAIII/artificial_intelligence_tutorial.pdf

7. https://www.emse.fr/ picard/cours/ai/chapter01.pdf

8. http://www.vssut.ac.in/lecture_notes/lecture1428643004.pdf

9. https://www.tutorialspoint.com/artificial_intelligence/artificial_intell igence_tutorial.pdf

10. https://www.cin.ufpe.br/ tfl2/artificial-intelligence-modernapproach.9780131038059.25368.pdf

\section{AUTHORS PROFILE}

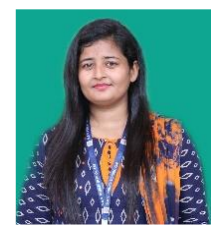

Banya Arabi Sahoo, Teacher at Dav public school, Chandrasekharpur, Bhubaneswar, Odisha in computer science dept. This is my first review paper 